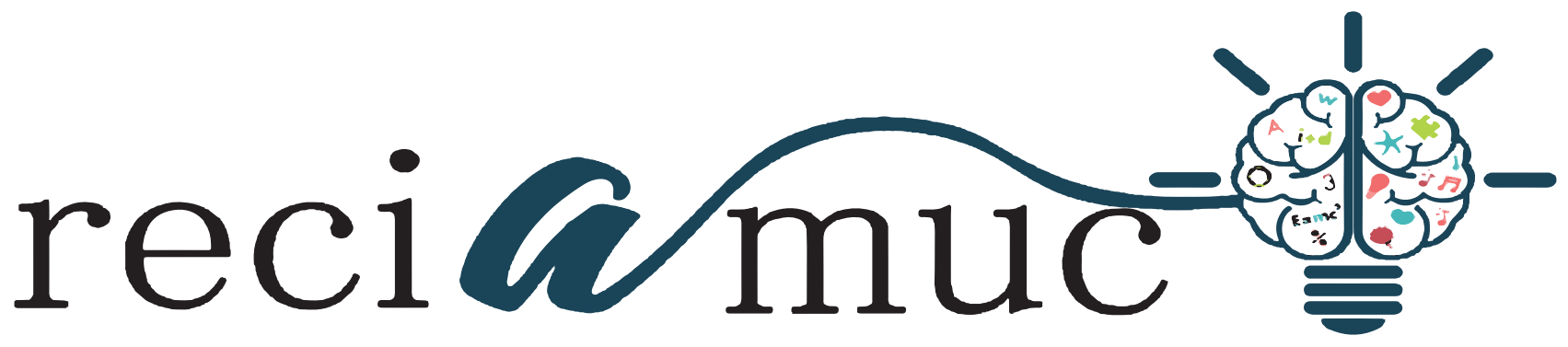

DOI: 10.26820/reciamuc/5.(4).noviembre.2021.196-205

URL: https://reciamuc.com/index.php/RECIAMUC/article/view/742

EDITORIAL: Saberes del Conocimiento

REVISTA: RECIAMUC

ISSN: 2588-0748

TIPO DE INVESTIGACIÓN: Artículo de Revisión

CóDIGO UNESCO: 32 Ciencias Médicas

PAGINAS: $196-205$

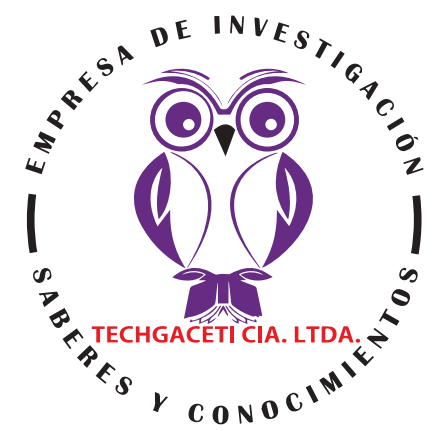

\title{
Incidencia de tromboembolia pulmonar masiva (TEP) en $\mathrm{UCI}$
}

Incidence of massive pulmonary embolism (PE) in ICU Incidência de embolia pulmonar maciça (EP) em UTI Jessica Ariana Guerra Fernández'; Carlos Andrés Villao Navas²; Sofia Alejandra Santos Benavides ${ }^{3}$

RECIBIDO: 15/09/2021 ACEPTADO: 05/10/2021 PUBLICADO: 29/11/2021

1. Magister en Seguridad y Salud Ocupacional; Médica; Investigador Independiente; Guayaquil, Ecuador; jeguerra.md@gmail.com; (D) https://orcid.org/0000-0002-1066-6610

2. Médico; Investigador Independiente; Guayaquil, Ecuador; andnav007@gmail.com; (D) https://orcid.org/00000002-2940-1225

3. Médico; Investigador Independiente; Guayaquil, Ecuador; sofilusantos@hotmail.com; (D) https://orcid.org/00000002-8497-0447

\section{CORRESPONDENCIA \\ Jessica Ariana Guerra Fernández \\ jeguerra.md@gmail.com \\ Guayaquil, Ecuador}

○ RECIAMUC; Editorial Saberes del Conocimiento, 2021 


\section{RESUMEN}

El tromboembolismo pulmonar (TEP) es una entidad clínico-patológica que se desencadena como consecuencia de la obstrucción arterial pulmonar por causa de un trombo desarrollado in situ o de otro material procedente del sistema venoso del resto del organismo. Esta enfermedad es uno de los problemas médicos más graves y comunes en la práctica diaria. Su importancia es tal que supera en estudios de necropsias a algunas enfermedades como las neumonías y el cáncer de pulmón. La metodología utilizada para el presente trabajo de investigación, se enfoca hacia una metodología orientada hacia la necesidad de indagar en forma precisa y coherente una situación. Enmarcada dentro de una revisión bibliográfica de tipo documental, ya que nos vamos a ocupar de temas planteados a nivel teórico como es Incidencia de tromboembolia pulmonar masiva (TEP) en UCl. La técnica para la recolección de datos está constituida por materiales impresos, audiovisuales y electrónicos, estos últimos como Google Académico, PubMed, entre otros. La información aquí obtenida será revisada para su posterior análisis. La incidencia de pacientes que ingresan a UCI, lógicamente está asociada a las complicaciones intrínsecas de esta patología, independientemente que la tromboembolia pulmonar sea provocada o no provocada. En este sentido en los casos clínicos aquí presentados y resumidos, solo en dos hacen referencia de casos en UCl, pero específicamente en un solo caso se especifica el porcentaje de $23,2 \%$ para 522 pacientes lo que esto indica es que es un número importante a tomar en cuenta. Entre otros aspectos importantes es que, dentro de las manifestaciones clínicas, la disnea es una de las más recurrentes y entre los factores de riesgo predomina el tabaquismo, la inmovilización, la hipertensión arterial y en muy bajos casos la recurrencia de esta patología.

Palabras clave: Embolo, Pulmonar, UCI, Anticoagulante, Disnea.

\section{ABSTRACT}

Pulmonary thromboembolism (PE) is a clinicopathological entity that is triggered as a consequence of pulmonary arterial obstruction due to a thrombus developed in situ or from other material originating from the venous system of the rest of the body. This disease is one of the most serious and common medical problems in daily practice. Its importance is such that it surpasses some diseases such as pneumonia and lung cancer in autopsy studies. The methodology used for this research work is focused on a methodology oriented towards the need to investigate a situation precisely and coherently. Framed within a bibliographic review of a documentary type, since we are going to deal with issues raised at a theoretical level such as Incidence of massive pulmonary thromboembolism (PE) in ICU. The technique for data collection is made up of printed, audiovisual and electronic materials, the latter such as Google Scholar, PubMed, among others. The information obtained here will be reviewed for further analysis. The incidence of patients admitted to the ICU is logically associated with the intrinsic complications of this pathology, regardless of whether the pulmonary embolism is provoked or unprovoked. In this sense, in the clinical cases presented and summarized here, only two refer to cases in the ICU, but specifically in a single case the percentage of $23.2 \%$ is specified for 522 patients, what this indicates is that it is an important number to take into account. Among other important aspects is that, within the clinical manifestations, dyspnea is one of the most recurrent and among the risk factors smoking, immobilization, arterial hypertension predominate and in very low cases the recurrence of this pathology.

Keywords: Embolism, Pulmonary, ICU, Anticoagulant, Dyspnea.

\section{RESUMO}

O tromboembolismo pulmonar (EP) é uma entidade clínico-patológica desencadeada em consequência da obstrução arterial pulmonar por trombo desenvolvido in situ ou por outro material proveniente do sistema venoso do resto do corpo. Esta doença é um dos problemas médicos mais graves e comuns na prática diária. Sua importância é tanta que ultrapassa algumas doenças como pneumonia e câncer de pulmão em estudos de autópsia. A metodologia utilizada para este trabalho de investigação centra-se numa metodologia orientada para a necessidade de investigar uma situação de forma precisa e coerente. Enquadrado dentro de uma revisão bibliográfica de tipo documental, uma vez que trataremos de questões levantadas a nível teórico como Incidência de tromboembolismo pulmonar maciço (EP) em UTI. A técnica de coleta de dados é composta por materiais impressos, audiovisuais e eletrônicos, estes últimos como Google Scholar, PubMed, entre outros. As informações aqui obtidas serão revisadas para análises posteriores. A incidência de pacientes internados em UTI está logicamente associada às complicações intrínsecas dessa patologia, independentemente de a embolia pulmonar ser provocada ou não. Nesse sentido, nos casos clínicos aqui apresentados e resumidos, apenas dois referem-se a casos em UTI, mas especificamente em um único caso especifica-se o percentual de 23,2\% para 522 pacientes, o que indica que se trata de um número importante para levar em consideração. Entre outros aspectos importantes está que, dentro das manifestações clínicas, a dispneia é uma das mais recorrentes e entre os fatores de risco predomina o tabagismo, a imobilização, a hipertensão arterial e em casos muito baixos a recorrência desta patologia.

Palavras-chave: Embolia Pulmonar, UTI, Anticoagulante, Dispnéia. 


\section{Introducción}

El tromboembolismo pulmonar (TEP) es una entidad clínico-patológica que se desencadena como consecuencia de la obstrucción arterial pulmonar por causa de un trombo desarrollado in situ o de otro material procedente del sistema venoso del resto del organismo. Los émbolos pulmonares proceden del sistema venoso profundo de los miembros inferiores. La trombosis venosa profunda (TVP) de miembros superiores supone del $1 \%$ al $4 \%$ de todos los casos de TVP, con una incidencia de tres casos por cada 100.000 habitantes por año (LARA CARCAMO, 2013, pág. 6). Si bien la prevalencia precisa de la ETV se desconoce, sabemos que la incidencia de TVP va desde 1 caso/10,000 adultos jóvenes a 1 caso/100 adultos mayores. En personas de 65 a 69 años la incidencia es de 1.8 casos/1,000 habitantes/año y aumenta a 3.1 casos/ 1,000 habitantes/año entre 85 y 89 años (Cabrera-Rayo \& Nellen-Hummel, 2007, pág. 3).

Esta enfermedad es uno de los problemas médicos más graves y comunes en la práctica diaria. Su importancia es tal que supera en estudios de necropsias a algunas enfermedades como las neumonías y el cáncer de pulmón. Se estima que el 3\% de todas las muertes ocurridas en los hospitales pueden atribuirse a embolias pulmonares, por lo que representa un factor importante de muerte; cuando esta entidad no es tratada la mortalidad puede llegar al $38 \%$. Algunos trabajos ponen de manifiesto la importancia del diagnóstico precoz en el tratamiento inmediato (González, Pérez, Peláez, Rodríguez, \& Basalto, 2015).

Este trombo se desprende y viaja en forma de émbolo a través del sistema circulatorio, hasta alcanzar el árbol arterial pulmonar, donde queda enclavado causando la interrupción del flujo sanguíneo pulmonar y la correspondiente isquemia del territorio irrigado por la arteria afectada. En caso de no diagnosticarse y tratarse de manera tem- prana, la mayoría de los fallecimientos por TEP se producen en las primeras horas sin apenas dar tiempo a iniciar el tratamiento ni a poner en marcha el proceso diagnóstico. El resto de los fallecimientos se deben a recurrencias de la embolia pulmonar en el mismo territorio en que tuvo lugar el primer episodio (Herrería Palacios, 2020, págs. 2-3).

Los factores de riesgo para desarrollar TEP están relacionados con uno o varios de los mecanismos etiopatogénicos de la enfermedad: estasis, lesión endotelial e hipercoagulabilidad. Esta normativa los clasifica como mayores o menores, en función de que su riesgo protrombótico sea alto o moderado-bajo, respectivamente. Cuando la ETEV se asocia a factores de riesgo desencadenantes se califica como provocada o secundaria. Cuando no concurren factores desencadenantes se denomina no provocada, espontánea o idiopática (Uresandi, y otros, 2013, pág. 535).

Tabla 1. Riesgo absoluto de TVP en pacientes hospitalizados.

\begin{tabular}{lc}
\hline Grupo de pacientes & Prevalencia de TVP (\%) \\
\hline Padecimiento médico & $10-20$ \\
Cirugía general & $15-40$ \\
Cirugía ginecológica mayor & $15-40$ \\
Cirugía urológica mayor & $15-40$ \\
Neurocirugía & $15-40$ \\
Evento vascular cerebral & $20-50$ \\
Artroplastía de cadera o rodilla & $40-60$ \\
Trauma mayor & $40-80$ \\
Pacientes de terapia intensiva & $10-80$ \\
\hline
\end{tabular}

Fuente: (Cabrera-Rayo \& Nellen-Hummel, 2007) 


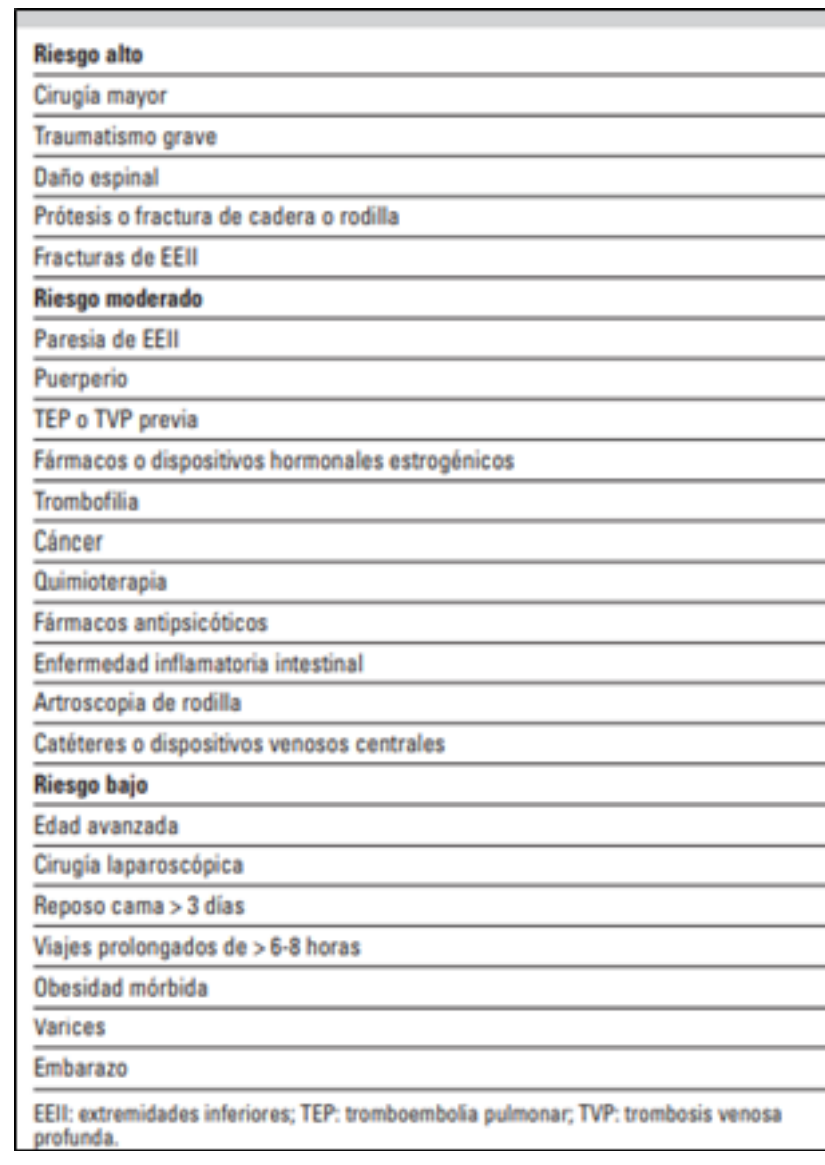

Imagen 1. Otra clasificación de factores de riesgo

Fuente: (Cabrera-Rayo \& Nellen-Hummel, 2007)

Desde el punto de vista epidemiológico, en el TEP, al igual que ocurre con otras patologías, se produce el fenómeno del iceberg: la punta del iceberg representa los casos de TEP diagnosticados y tratados partiendo o no de la sospecha clínica. La parte no visible del iceberg incluye los casos de TEP sospechados y no sospechados a partir de la clínica que presenta el paciente y que no han sido diagnosticados y, tampoco tratados. Este segundo grupo es mayoritario en relación al primero, dado que solamente una pequeña proporción de los casos de TEP se diagnostican y se tratan (Herrería Palacios, 2020, pág. 3).

\section{Manifestaciones clínicas}

- La presentación clínica puede ser muy variable y presentarse desde un shock o hipotensión sostenida a disnea leve. Puede incluso ser asintomática y diagnosticarse mediante pruebas de imagen realizadas para otros fines. El 30\% de los casos, el TEP tiene lugar en ausencia de factor predisponente alguno (TEP idiopático o no provocado). La gravedad de los síntomas depende principalmente de la magnitud del embolismo y la condición cardiorrespiratoria previa.

- En el TEP periférico se produce un infarto pulmonar que se manifiesta como irritación pleural y dolor tipo pleurítico hasta en $50 \%$ de los pacientes a veces acompañada de tos y hemoptisis.

- El síncope es raro, pero es una presentación importante de TEP en un 10\%, ya que puede ser indicio de una reducción grave de la reserva hemodinámica. La presencia de éste acompañado de shock cardiogénico generalmente obedecen a una tromboembolia pulmonar masiva (> 40\% de obstrucción de los vasos pulmonares) o también puede presentarse en un paciente con una TEP submasivo con función cardiaca limítrofe, lo cual augura mal pronóstico (Solórzano, 2015, pág. 32). 
Tabla 2. Diagnóstico

\begin{tabular}{|c|c|}
\hline Gasometría arterial & $\begin{array}{l}\text { El } 20 \% \text { de los casos cursan con normoxemia. La alcalosis respiratoria } \\
\text { asociada a hipoxemia arterial es el hallazgo más frecuente en el contexto } \\
\text { de un cuadro agudo }\end{array}$ \\
\hline Radiografía de tórax & $\begin{array}{l}\text { Puede mostrar signos que sugieren su existencia, pero son inespecíficos } \\
\text { como el aumento en el tamaño de la arteria pulmonar descendente derecha } \\
\text { (signo de Palla), imágenes de atelectasia, oligohemia focal (signo de } \\
\text { westermark), una densidad cuneiforme periférica por arriba del diafragma } \\
\text { (giba de Hampton) }\end{array}$ \\
\hline Ecocardiografía & $\begin{array}{l}\text { Puede ser normal hasta en el } 30 \% \text { de los pacientes. El hallazgo más } \\
\text { frecuente es la taquicardia sinusal. Es de gran utilidad para descartar } \\
\text { trastornos semejantes como el IAM, taponamiento pericárdico o disección } \\
\text { de aorta }\end{array}$ \\
\hline Determinación del dímero-D & $\begin{array}{l}\text { Muestra valores altos en TEP en virtud de la degradación de la fibrina } \\
\text { formados por plasmina durante la fibrinólisis, lo que normalmente ocurre } \\
\text { una hora después de la formación del trombo. El valor de corte para } \\
\text { considerar la prueba como positiva depende del método de determinación } \\
\text { utilizado. }\end{array}$ \\
\hline Angiografía pulmonar & $\begin{array}{l}\text { Es el estudio estándar de oro para el diagnóstico de TEP los inconvenientes } \\
\text { son que es invasiva, costosa, requiere de personal especializado para su } \\
\text { realización y no está exenta de complicaciones, está contraindicada en } \\
\text { insuficiencia renal o en pacientes gravemente enfermos }\end{array}$ \\
\hline Tomografía helicoidal contrastada & $\begin{array}{l}\text { Alternativa diagnóstica, con una sensibilidad y especificidad hasta del } \\
94 \% \text { en manos experimentadas. Es excelente para identificar émbolos en } \\
\text { vasos principales y lobares, su problema es que es incapaz para detectar } \\
\text { émbolos pequeños }\end{array}$ \\
\hline Resonancia magnética & $\begin{array}{l}\text { Herramienta diagnóstica prometedora especialmente en aquellos pacientes } \\
\text { con alergia al contraste o que deben de evitar el uso de radiaciones } \\
\text { ionizantes, el estudio positivo tiene un } 89 \% \text { de posibilidades de que esté } \\
\text { cursando con TEP sobre todo cuando se trata de obstrucción de vasos } \\
\text { grandes }\end{array}$ \\
\hline Ecocardiograma & $\begin{array}{l}\text { Alternativa diagnóstica para algunos. En los pacientes aparentemente } \\
\text { menos graves aporta una información pronostica importante sobre la } \\
\text { potencial gravedad del cuadro }\end{array}$ \\
\hline
\end{tabular}

Fuente: Elaboración Propia. Tomado de (Solórzano, 2015)

\begin{tabular}{|c|c|c|c|}
\hline \multicolumn{3}{|l|}{ Escala de Wells } & Puntuación \\
\hline \multicolumn{3}{|c|}{ Diagnóstico alternativo menos probable que la TEP } & 3,0 \\
\hline \multicolumn{3}{|l|}{ Sintomas o signos de TVP } & 3,0 \\
\hline \multicolumn{3}{|l|}{ Antecedentes de TEP o TVP } & 1,5 \\
\hline \multicolumn{3}{|c|}{ Inmovilización de al menos 3 dias o cirugia en el último mes } & 1,5 \\
\hline \multicolumn{3}{|c|}{ Frecuencia cardiaca $>100 /$ minutos } & 1.5 \\
\hline \multicolumn{3}{|l|}{ Hemoptisis } & 1,0 \\
\hline \multicolumn{3}{|c|}{ Cáncer en tratamiento activo o paliativo en los últimos 6 meses } & 1,0 \\
\hline & $\begin{array}{l}\text { Para dimero D } \\
\text { muy sensible }\end{array}$ & & $\begin{array}{l}\text { Para dimero D } \\
\text { menos sensible }\end{array}$ \\
\hline $\begin{array}{l}\text { Baja probabilidad } \\
\text { Intermedia probabilidad } \\
\text { Alta probabilidad }\end{array}$ & $\begin{array}{l}<2 \text { puntos } \\
2 \cdot 6 \text { puntos } \\
26 \text { puntos }\end{array}$ & $\begin{array}{l}\text { TEP poco probable } \\
\text { TEP probable }\end{array}$ & $\begin{array}{l}\$ 4 \text { puntos } \\
>4 \text { puntos }\end{array}$ \\
\hline \multicolumn{3}{|l|}{ Escala de Ginebra } & Puntuación \\
\hline \multicolumn{3}{|l|}{ Edad $>65$ anos } & 1,0 \\
\hline \multicolumn{3}{|l|}{ Antecedente de TVP o TEP } & 3,0 \\
\hline \multicolumn{3}{|c|}{ Cirugia con anestesia general o fractura $\leq 1$ mes } & 2,0 \\
\hline \multicolumn{3}{|c|}{ Cáncer activo sólido o hematológico o curado $\leq 1$ año } & 2,0 \\
\hline \multicolumn{3}{|l|}{ Dolor unilateral en EEII } & 3,0 \\
\hline \multicolumn{3}{|l|}{ Hemoptisis } & 2,0 \\
\hline \multicolumn{3}{|c|}{ Frecuencia cardiaca $75-94 /$ minuto } & 3,0 \\
\hline \multicolumn{3}{|c|}{ Frecuencia cardiaca $295 /$ minuto } & 5,0 \\
\hline \multicolumn{3}{|c|}{ Dolor a la palpación en EEll y edema unilateral } & 4,0 \\
\hline \multicolumn{4}{|c|}{$\begin{array}{l}\text { Para dimero D } \\
\text { muy sensible }\end{array}$} \\
\hline $\begin{array}{l}\text { Baja probabilidad } \\
\text { Intermedia probabilidad } \\
\text { Alta probabilidad }\end{array}$ & $\begin{array}{l}0-3 \text { puntos } \\
4-10 \text { puntos } \\
211 \text { puntos }\end{array}$ & & \\
\hline
\end{tabular}

Imagen 2. Escalas de puntuación para la graduación de la probabilidad clínica en la tromboembolia de pulmón aguda sintomática

Fuente: (Jiménez, Kopecna, de Miguel, \& Sueiro, 2014) 


\section{Tromboembolia pulmonar y Covid 19}

Las manifestaciones trombóticos pulmonares se ha descrito en autopsias de pacientes infectados en las epidemias por los coronavirus SARS-CoV y MERS-CoV en 2003 y 2012, respectivamente. La fisiopatología que relaciona el TEP con la infección por SARS-CoV-2 no se conoce bien y parece estar relacionada con un estado de hipercoagulabilidad. Estudios recientes han demostrado que niveles de dímero $D$ superiores a $1.000 \mathrm{ng} / \mathrm{ml}$ en pacientes con COVID-19 constituyen un índice pronóstico relevante de mortalidad (Franco-Moreno, Muñoz-Rivas, Mestre-Gómez, \& Torres-Macho, 2020, pág. 459).

\begin{tabular}{|c|c|c|c|c|c|}
\hline Estudio & $\begin{array}{l}\text { Número de } \\
\text { pacientes }\end{array}$ & $\begin{array}{l}\text { Ámbito del } \\
\text { estudio }\end{array}$ & $\begin{array}{l}\text { Incidencia de } \\
\operatorname{TEV}(\mathrm{n} ; \mathrm{\%})\end{array}$ & $\begin{array}{l}\text { Régimen de terapia } \\
\text { antitrombótica }\end{array}$ & Tipo de evento \\
\hline$\overline{\text { Klok et at.' }}$ & 184 & UCI & $26(14,13)$ & Heparina profiláctica & $\begin{array}{l}25 \text { pacientes con TEP } \\
\text { Un paciente con TVP } \\
\text { Otros: un TVP de extremidad } \\
\text { superior asociada a catéter } \\
\text { y } 3 \text { ictus }\end{array}$ \\
\hline Uitjos et al. ${ }^{2}$ & 26 & UCI & $\begin{array}{l}18 \text { (69) para } \\
\text { trombosis } \\
\text { venosa de EEll y } \\
6 \text { (23) para TEP }\end{array}$ & $\begin{array}{l}8 \text { pacientes heparina } \\
\text { profiláctica } \\
18 \text { pacientes heparina } \\
\text { terapéutica }\end{array}$ & $\begin{array}{l}6 \text { pacientes con TEP } \\
14 \text { pacientes con TVP } \\
4 \text { pacientes con TVS }\end{array}$ \\
\hline Cui et al. ${ }^{3}$ & 81 & UCI & $20(25)$ & No administrado & 20 pacientes con TVP \\
\hline Poissy et al. ${ }^{4}$ & 107 & UCI & $\begin{array}{l}22(20,6) \text { para } \\
\text { TEP y } 5(4,7) \\
\text { para TVP }\end{array}$ & $\begin{array}{l}20 \text { pacientes heparina } \\
\text { profiláctica } \\
\text { Un paciente AVK } \\
\text { Un paciente heparina } \\
\text { terapéutica }\end{array}$ & $\begin{array}{l}22 \text { pacientes con TEP; } \\
3 \text { pacientes con TVP } \\
\text { concomitante }(13,6 \%) \\
5 \text { pacientes con TVP }\end{array}$ \\
\hline Lodigiani et at. ${ }^{5}$ & 388 & UCI y UH & $16(4,4)$ & $\begin{array}{l}100 \% \text { de pacientes de } \\
\text { UCI heparina profiláctica } \\
75 \% \text { de pacientes de UH } \\
\text { heparina profiláctica }\end{array}$ & $\begin{array}{l}10 \text { pacientes con TEP; } \\
1 \text { paciente con TVP } \\
\text { concomitante (10\%) } \\
4 \text { pacientes con TVP proximal } \\
\text { Un paciente con TVP distal } \\
\text { Otros: } 1 \text { TVP de extremidad } \\
\text { superior asociada a catéter, } \\
9 \text { ictus y } 4 \text { SCA }\end{array}$ \\
\hline Helms et al. ${ }^{6}$ & 150 & UCI & $\begin{array}{l}25(16,7) \text { para } \\
\text { TEP y } 3(2) \\
\text { para TVP }\end{array}$ & $\begin{array}{l}\text { Heparina } \\
\text { profiláctica }\end{array}$ & $\begin{array}{l}25 \text { pacientes con TEP } \\
3 \text { pacientes con TVP } \\
\text { Otros: } 2 \text { ictus, una isquemia } \\
\text { mesentérica y una isquemia } \\
\text { arterial periférica }\end{array}$ \\
\hline Middeldorp et al. ${ }^{7}$ & 198 & UCI y UH & $39(20)$ & $\begin{array}{l}\text { Heparina } \\
\text { profiláctica }\end{array}$ & $\begin{array}{l}13 \text { pacientes con TEP } \\
14 \text { pacientes con TVP proximal } \\
11 \text { pacientes con TVP distal } \\
\text { Un paciente con TVP de } \\
\text { extremidad superior }\end{array}$ \\
\hline
\end{tabular}

Imagen 3. Series de casos de pacientes con COVID-19 y TEV.

Fuente: (Franco-Moreno, Muñoz-Rivas, Mestre-Gómez, \& Torres-Macho, 2020, pág. 459).

\section{Complicaciones a largo plazo de la trom- boembolia pulmonar}

1. Síndrome postrombótico: Se denomina síndrome postrombótico al desarrollo de síntomas y signos de insuficiencia venosa crónica después de una TVP. El término postrombótico sustituye a la denominación previa de síndrome postflebítico.
Estudios prospectivos con seguimiento a largo plazo indican que entre un 20 y un $50 \%$ de los pacientes con una TVP presenta como secuela un síndrome postrombótico. Entre un 5 y un $10 \%$ de los pacientes desarrolla un cuadro grave que puede conllevar la aparición de úlceras. 
2. Hipertensión pulmonar tromboembólica crónica: La complicación más temida a largo plazo del TEP es la hipertensión pulmonar tromboembólica crónica (HPTEC) La HPTEC se define por la existencia de una presión media de la arteria pulmonar $\geq 25 \mathrm{mmHg}$ medida mediante cateterismo cardíaco derecho, con una presión capilar pulmonar $\leq 15 \mathrm{mmHg}$, en presencia de trombo crónico/organizado en las arterias pulmonares (principal, lobar, segmentaria o subsegmentaria), después de al menos tres meses de correcta anticoagulación. Los estudios prospectivos sugieren que la HPTEC ocurre aproximadamente en un 3\% de los pacientes tratados por un TEP.

3. Recurrencia de la ETEV: La ETEV recidiva frecuentemente. La presencia de un factor de riesgo transitorio o persistente, el desarrollo de una ETEV en el contexto de un cáncer activo o la naturaleza no provocada de la ETEV, son los principales factores que determinan el riesgo de recurrencia de la ETEV tras la suspensión de la anticoagulación. Entre los pacientes con ETEV provocada por un factor de riesgo reversible, el riesgo de recurrencia es menor si el factor es cirugía reciente comparado con un desencadenante no quirúrgico (por ejemplo, tratamiento estrogénico, embarazo, trauma en miembro inferior o vuelo de 6 horas) (Fidalgo Fernández, 2020, págs. 34-35).

\section{Tratamiento de la tromboembolia de pul- món en fase aguda}

El tratamiento de la fase aguda de la TEP tiene como objetivos la estabilización médica del paciente, el alivio de los síntomas y la prevención de las recurrencias. En la mayoría de las ocasiones, el tratamiento anticoagulante convencional es suficiente para evitar la progresión del trombo, mientras el sistema fibrinolítico endógeno resuelve la obstrucción vascular y se desarrolla la circulación colateral. En una minoría de pacientes, habitualmente aquellos con inestabilidad hemodinámica (TEP de alto riesgo) o contraindicación para la anticoagulación, se requieren otros tratamientos farmacológicos (trombolíticos) o medidas mecánicas (filtros de vena cava) para acelerar la lisis del coágulo o prevenir su embolización a los pulmones (Jiménez, Kopecna, de Miguel, \& Sueiro, 2014, pág. 4054).

Tabla 2. Tratamiento tromboembolia de pulmón en fase aguda.

\begin{tabular}{|c|l|}
\hline $\begin{array}{c}\text { Anticoagulación } \\
\text { parenteral }\end{array}$ & $\begin{array}{l}\text { Para el tratamiento agudo de la mayoría de los pacientes con TEP se prefiere el uso de } \\
\text { HBPM subcutánea o de fondaparinux subcutáneo a la HNF, ya que se asocian a un riesgo } \\
\text { menor de sangrados mayores y de trombocitopenia inducida por heparina. Actualmente } \\
\text { la HNF se reserva para pacientes en los que se considera la utilización de tratamiento } \\
\text { fibrinolítico (TEP de riesgo intermedio o alto), y para pacientes con alto riesgo de } \\
\text { sangrado que van a ser anticoagulados. Para pacientes con insuficiencia renal grave } \\
\text { (aclaramiento de creatinina menor de 30 ml/min) se puede utilizar HNF o HBPM en las } \\
\text { dosis recomendadas en cada ficha técnica. }\end{array}$ \\
\hline $\begin{array}{c}\text { Antagonistas de la } \\
\text { vitamina K }\end{array}$ & $\begin{array}{l}\text { Los AVK deben ser administrados lo antes posible, preferiblemente el primer día } \\
\text { después del diagnóstico. Se deben } \\
\text { solapar con la anticoagulación parenteral durante un mínimo } \\
\text { de } 5 \text { días, y hasta que el cociente normalizado internacional } \\
\text { (INR) esté entre 2 y 3 durante dos días consecutivos. }\end{array}$ \\
\hline Dabigatrán & $\begin{array}{l}\text { Es un inhibidor directo de la trombina. En el análisis combinado de dos ensayos clínicos } \\
\text { para TVP y para TEP (22\%), dabigatrán no fue inferior a warfarina en términos de } \\
\text { eficacia (recurrencias trombóticas o muerte por ETEV) (HR 1,09; IC 95\%; 0,76-1,57) o } \\
\text { de seguridad (sangrados mayores) (HR 0,73; IC 95\%; 0,48-1,11). Se administra por vía } \\
\text { oral, en dosis de 150 mg dos veces al día, tras un período de } 5 \text { a 10 días de } \\
\text { anticoagulación parenteral }\end{array}$ \\
\hline
\end{tabular}




\begin{tabular}{|c|c|}
\hline Rivaroxabán & $\begin{array}{l}\text { Es un inhibidor directo y selectivo del factor Xa. En el ensayo clínico EINSTEIN-PE, } \\
\text { rivaroxabán se asoció a una eficacia similar a la del tratamiento estándar (HR 1,12; IC } \\
95 \% ; 0,75-1,68 \text { ), la misma seguridad (sangrados totales) (HR 0,90; IC 95\%; 0,76-1,07), } \\
\text { y una reducción a la mitad en las hemorragias graves (HR 0,49; IC 95\%; 0,31-0,79). Se } \\
\text { administra por vía oral, en dosis de } 15 \text { mg dos veces al día durante } 3 \text { semanas seguido } \\
\text { de } 20 \text { mg una vez al día }\end{array}$ \\
\hline Apixabán & $\begin{array}{l}\text { Ha sido evaluado en un ensayo clínico para el tratamiento de pacientes con TVP o TEP } \\
\text { (34\%) durante los } 6 \text { primeros meses después del evento trombótico. Comparado con el } \\
\text { tratamiento estándar, apixabán demostró una eficacia similar (riesgo relativo [RR] 0,84; } \\
\text { IC 95\%; 0,60-1,18), y una reducción estadísticamente significativa de las hemorragias } \\
\text { graves (RR } 0,31 ; \text { IC 95\%; } 0,17-0,55 \text { ) y de las hemorragias no graves clínicamente } \\
\text { relevantes (RR 0,48; IC 95\%;0,38-0,60). Se administra por vía oral, en dosis de } 10 \mathrm{mg} \\
\text { dos veces al día los primeros } 7 \text { días, seguido de } 5 \mathrm{mg} \text { dos veces al día. }\end{array}$ \\
\hline Edox & $\begin{array}{l}\text { Fue comparado con el tratamiento convencional en } 8.240 \text { pacientes con ETEV ( } 3.319 \\
\text { con TEP) } 28 \text {. Edoxabán no fue inferior a warfarina para el evento primario de eficacia } \\
\text { (recurrencia trombótica sintomática o TEP fatal) (HR } 0,89 ; \text { IC } 95 \% ; 0,70-1,13 \text { ). El } \\
\text { evento primario de seguridad (sangrado mayor o sangrado no mayor clínicamente } \\
\text { relevante) ocurrió con menos frecuencia en el grupo de pacientes que recibió edoxabán } \\
\text { (HR } 0,81 ; \mathrm{IC} 95 \% ; 0,71-0,94 \text { ). Se administra por vía oral en dosis de } 60 \mathrm{mg} \text { una vez al } \\
\text { día }(30 \mathrm{mg} \text { al día en caso de aclaramiento de creatinina } 30-50 \mathrm{ml} / \mathrm{min} \text { o peso corporal } \\
\text { menor de } 60 \mathrm{~kg} \text { ), tras un período de } 5 \text { a } 10 \text { días de anticoagulación parenteral. }\end{array}$ \\
\hline $\begin{array}{c}\text { Tratamiento } \\
\text { domiciliario o alta } \\
\text { precoz frente al alta } \\
\text { estándar en pacientes } \\
\text { con tromboembolia de } \\
\text { pulmón } \\
\end{array}$ & $\begin{array}{l}\text { Se podría considerar el tratamiento ambulatorio o el alta precoz de pacientes con TEP } \\
\text { que cumplan los siguientes requisitos: a) clínicamente estables con buena reserva cardio } \\
\text { pulmonar, y una escala clínica validada de bajo riesgo (por ejemplo, PESI o PESIs); b) } \\
\text { buen soporte social con rápido acceso a atención médica y c) cumplimentación esperable } \\
\text { del tratamiento }\end{array}$ \\
\hline
\end{tabular}

Fuente: Elaboración Propia. Tomado de (Jiménez, Kopecna, de Miguel, \& Sueiro, 2014).

\section{Metodología}

La metodología utilizada para el presente trabajo de investigación, se enfoca hacia una metodología orientada hacia la necesidad de indagar en forma precisa y coherente una situación. Enmarcada dentro de una revisión bibliográfica de tipo documental, ya que nos vamos a ocupar de temas planteados a nivel teórico como es Incidencia de tromboembolia pulmonar masiva (TEP) en UCl. La técnica para la recolección de datos está constituida por materiales impresos, audiovisuales y electrónicos, estos últimos como Google Académico, PubMed, entre otros. La información aquí obtenida será revisada para su posterior análisis.

\section{Resultados}

\section{Incidencia}

1. Díaz Santos \& Blanco Mosquera (2015),en su trabajo de investigacion, analizaron a 76 pacientes masculino o femenino, mayores de 18 años de edad con sospecha diagnostica de embolia pulmonar que haya requerido hospitalización en la unidad de cuidados intensivos. Entre los resultados mas importantes de estudio se destaca lo siguiente:

- El 50\% de los pacientes que ingresaron a UCl están entre los 61-80 años de edad, un 14,5\% mayores de 80 años, $27,6 \%$ entre los 41-60 años y 7,9\% menos de 40 años.

- Entre los factores de riesgo más destacados en los pacientes con embolia pulmonar 53,9\% tabaquismo, 39,5\% hipertensión arterial, 35,5\% EPOC, 23,7\% falla cardiaca, la hipertensión pulmonar aparece como factor diagnostico en el 6 puesto con un $18,4 \%$.

- Entre los mayores síntomas se destacan: $78,9 \%$ disnea, 40,8\% taquicardia y $36,8 \%$ dolor torácico.

- 32 pacientes fallecieron, como consecuencia de shock al ingreso 35\%, shock durante la hospitalización 22,2\% y un $28,6 \%$ por paro cardiorrespiratorio.

2. LARA CARCAMO (2013), en su trabajo de investigacion analizaron a 15 pacientes, 
de los cuales 6 estan diagnsticados con trombombolia pulmonar y 9 sin diagnostico. En este contexto solo analizaremos los dagnosticados, ingresados al servicios de terapia intensiva. Entre los resultados se tienen:

El $50 \%$ de los pacientes diagnosticados presentaron como factores de riesgo antecedentes de ETEV, el 83,3\% cirugía/ inmovilización y un 16,67\% trombofilia.

- En cuanto a la sintomatología todos presentaron disnea, taquicardia y dolor torácico. Un 83,3\% taquipnea, entre otros.

- En base a la clasificación de Wells, 1 paciente presento probabilidad clínica baja de desarrollar embolia pulmonar, 3 pacientes presentaron probabilidad clínica moderada de desarrollar embolia pulmonar y 2 pacientes presentaron probabilidad clínica alta de desarrollar embolia pulmonar.

- 5 pacientes presentaron en sus exámenes de laboratorio valores de dímero $D$ positivos.

3. Stewart Savage (2021), en su trabajo de investigacion, analiza a 522 pacientes diagnsoticados con tromboembelismo pulmonar, entre los resultados principales sedestacan:

- El $26 \%$ de los pacientes tienen edades comprendidas entre los 60 - 69 años, un $15 \%$ con edades entre los 50 a 59 años, $15 \%$ entre los 70 a 79 años y $13 \%$ mayores a 80 años.

- Entre los factores de riesgo asociados con la patología, el 47,4\% inmovilización, 36,7\% TVP, cirugía previa 27,3\%, cáncer $21,5 \%$ y tabaquismo $20 \%$, por mencionar las más relevantes.

- Un 5,2\% presento tromboembolia pulmonar previa, de los cuales el 37\% tenía 2 factores de riesgo, el $28 \%$ un factor de riesgo y 21\% más de 3 factores de riesgo.
- La sintomatología presentada por los pacientes en un $85 \%$ disnea, $50 \%$ dolor torácico, taquipnea $34,9 \%$ y palpitaciones $30,1 \%$.

- Complicaciones presentadas, 27,4\% hipertensión arterial pulmonar, $25,1 \%$ insuficiencia respiratoria, $23,2 \%$ ingresaron a $\mathrm{UCI}, 20,2 \%$ fallecieron, entre otros.

- El 97,9\% de los pacientes recibieron como tratamiento anticoagulante HBPM.

\section{Conclusiones}

La incidencia de pacientes que ingresan a $\mathrm{UCl}$, lógicamente está asociada a las complicaciones intrínsecas de esta patología, independientemente que la tromboembolia pulmonar sea provocada o no provocada. En este sentido en los casos clínicos aquí presentados y resumidos, solo en dos hacen referencia de casos en $\mathrm{UCl}$, pero específicamente en un solo caso se especifica el porcentaje de 23,2\% para e522 pacientes lo que esto indica es que es un número importante a tomar en cuenta. Entre otros aspectos importantes es que, dentro de las manifestaciones clínicas, la disnea es una de las más recurrentes y entre los factores de riesgo predomina el tabaquismo, la inmovilización, la hipertensión arterial y en muy bajos casos la recurrencia de esta patología.

\section{Bibliografía}

Cabrera-Rayo, A., \& Nellen-Hummel, H. (2007). Epidemiología de la enfermedad tromboembólica venosa. Gaceta Médica de México, 143(S1), 3-5.

Díaz Santos, G. A., \& Blanco Mosquera, J. (2015). Análisis de la sobrevida en los pacientes con diagnóstico de tromboembolismo pulmonar que requirieron atención en la unidad de cuidado intensivo en el Hospital Santa Clara de Bogotá, entre 2005 al 2014.

Fidalgo Fernández, M. Á. (2020). Características clínicas y biológicas del tromboembolismo pulmonar no provocado. Estudio de las mutaciones somáticas relacionadas con la hematopoyesis clonal de significado intermedio. 
Franco-Moreno, A., Muñoz-Rivas, N., Mestre-Gómez, B., \& Torres-Macho, J. (2020). Tromboembolismo pulmonar y COVID-19: un cambio de paradigma. Revista clínica española, 220(7), 220(7), 459. doi:https://dx.doi.org/10.1016\%2Fj.rce.2020.05.006

González, I. B., Pérez, R., Peláez, R., Rodríguez, C., \& Basalto, M. (2015). Incidencia del tromboembolismo pulmonar en el hospital Manuel Ascunce Domenech. Archivo Médico Camagüey, 6(7).

Herrería Palacios, P. (2020). TEP no sospechado en Urgencias.

Jiménez, D., Kopecna, D., de Miguel, J., \& Sueiro, A. (2014). Tromboembolia de pulmón. Medicine-Programa de Formación Médica Continuada Acreditado, 11(68), 4051-4058. doi:https://doi.org/10.1016/ S0304-5412(14)70882-0

LARA CARCAMO, V. (2013). ROMBOEMBOLIA PULMONAR: RELACIÓN ENTRE PROBABILIDAD CLÍNICA Y LOS NIVELES SÉRICOS DEL DÍMERO-D EN PACIENTES DE LA UNIDAD DE TERAPIA INTENSIVA DEL HOSPITAL DE GINECOLOGÍA Y OBSTETRICIA, EN EL PERIODO ENERO 2010 -NOVIEMBRE 2012.
Solórzano, A. V. (2015). Tromboembolismo pulmonar (TEP). Revista Médica de Costa Rica y Centroamérica, 71(614), 31-36.

Stewart Savage, S. S. (2021). Características clínicas de los pacientes atendidos en el Hospital San Juan de Dios con diagnóstico de treomboembolismo pulmonar hospitalizados en el periodo enero del 2015 a diciembre del 2020.

Uresandi, F., Monreal, M., García-Bragado, F., Domenech, P., Lecumberri, R., Escribano, P., \& Jiménez, D. (2013). Consenso nacional sobre el diagnóstico, estratificación de riesgo y tratamiento de los pacientes con tromboembolia pulmonar. Arch Bronconeumol, 49(12), 534-47. doi:http://dx.doi. org/10.1016/j.arbres.2013.07.008

\section{CITAR ESTE ARTICULO:}

Guerra Fernández, J. A., Villao Navas, C. A., \& Santos Benavides, S. A. (2021). Incidencia de tromboembolia pulmonar masiva (TEP) en UCI. RECIAMUC, 5(4), 196-205. https://doi.org/10.26820/reciamuc/5.(4).noviembre.2021.196-205

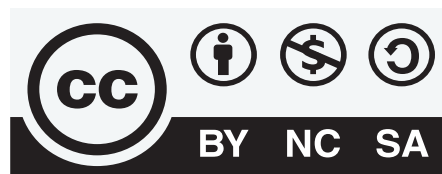

CREATIVE COMMONS RECONOCIMIENTO-NOCOMERCIAL-COMPARTIRIGUAL 4.0. 